J. Perinat. Med. 15 (1987) 435

\title{
Pregnancy-related factors and diseases influencing surfactant
}

\author{
Martin J. Whittle and H. Gordon Dobbie
}

Department of Midwifery, The Quenn Mother's Hospital, Yorkhill, Glasgow, Scotland

\section{Introduction}

For many years obstetricians have had the impression that certain pregnancy complications appear to be associated with an unusually low incidence of neonatal respiratory distress syndrome (RDS). Why this should be is unclear but it was proposed that "fetal stress" arising from a hostile intrauterine environment was a likely underlying factor. The conditions associated with accelerated lung maturity are

- hypertension,

- renal disease,

- antepartum hemorrhage,

- preterm rupture of membranes,

- intrauterine growth retardation.

The effect of diabetes mellitus on the incidence of RDS also remains undecided but in general it has been suggested that this condition may delay or alter surfactant production.

Unfortunately the problems of neonatal respiratory disease and the influence of obstetric factors are complicated by a number of issues. Firstly the best indicator of mature fetal/neonatal lungs must be decided. Should it be the presence of adequate surfactant levels in the amniotic fluid/pharyngeal aspirate or should the neonatal respiratory performance be of greater importance? Secondly, how certainly can a diagnosis of RDS be made and are the criteria in current use relevant? Thirdly how important are other factors, such as mode of delivery, condition at birth and the vigor of the neonatal resuscitation, in influencing neonatal functional maturity? Finally, how is it possible to derive a "normal" timetable for fetal lung maturity using prematurely born babies who, by definition, are not normal either because of the time of their birth or underlying maternal pathology.

\section{Curriculum vitae}

Dr MARTIN WhitTLe qualified MB ChB 1972, University of Manchester, United Kingdom and became Member of the Royal College of Obstetricians/ Gynaecologists in 1977. He has had a long-standing interest in surfactant and the effect of pregnancy complications on neonatal RDS. He was awarded an

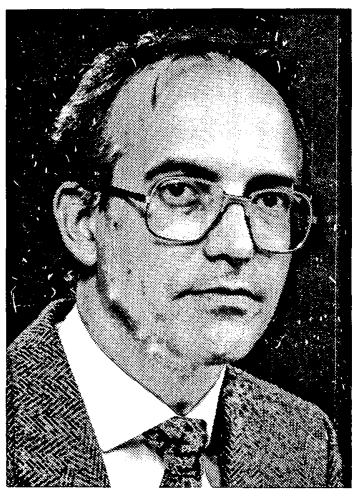
MD in 1980. Since 1982 he has been Consultant Obstetrician at the Queen Mother's Hospital, Glasgow, with special responsibilities in perinatal medicine.

Biochemical methods to evaluate amniotic fluid for evidence of fetal lung maturity have been in use for a number of years. In a study completed a few years ago [5] we demonstrated that, in the presence of preterm rupture of the membranes (PROM), a significantly greater proportion of amniotic fluids contained the surfactant, phosphatidylglycerol (PG). Other "stressful conditions" however did not show this phenomenon suggesting that PROM produced a specific stimulus to surfactant production.

Why should this be? We established a hypothesis that the fetal stress products, cortisol and the catecholamines, may affect surfactant production and release, to produce optimal lung function. A prospective study was undertaken using pregnancies which terminated at or before 35 weeks [2]. 


\section{Material and methods}

A total of 189 babies was studied in an attempt to correlate obstetric complications with outcome and a number of biochemical parameters including amniotic/pharyngeal fluid surfactant and cord blood artery and vein cortisol, adrenaline, noradrenaline and gases. The complications studied were hypertension/proteinuria, vaginal bleeding, preterm labor/ruptured membranes, abnormal antenatal cardiotocography and small for gestational age.

Amniotic/pharyngeal fluids were collected whenever possible. Some of the amniotic fluids were collected from the vagina and it was not always possible to obtain material within 72 hours of delivery. Amniotic/pharyngeal fluids were an-

Table I. Preterm baby study - overall data.

\begin{tabular}{lllll}
\hline $\begin{array}{l}\text { Gestational age } \\
\text { (wks) }\end{array}$ & N & $\begin{array}{l}\text { B.W. } \\
(\mathrm{kg})\end{array}$ & $\begin{array}{l}\text { RDS } \\
(\%)\end{array}$ & $\begin{array}{l}\text { Death } \\
(\%)\end{array}$ \\
\hline $24-29$ & 46 & 1.09 & 78 & 43 \\
$30-33$ & 74 & 1.72 & 34 & 5 \\
34,35 & 69 & 2.16 & 16 & 4 \\
\hline
\end{tabular}

189 alyzed by two-dimensional thin layer chromatography (2-DTLC) technique [6] and when possible the lecithin and phosphatidylglycerol was quantified from the phosphate concentration measured in the eluted spots [3].

Cortisol levels were measured using a radioimmune assay kit (Amersham International, UK) and the catecholamines were assayed by a radioenzymatic method [1]. Blood $\mathrm{pH}$ was measured in the cord bloods using standard laboratory equipment.

The non-parametric statistics involved chisquared analysis and differences between means was determined using Student's t test.

\section{Results}

Table I shows the overall results for the study. Tables II, III and IV show the effect of the obstetric complications on outcome for three arbitrarily selected gestational ages groups. There are more numbers here because some pregnancies were complicated by more than one problem and it was often impossible to assign one major complication to a single case. Nevertheless, none of the complications appear to have any striking effect on the incidence of RDS, death or the presence of PG.

Table II. Effect of obstetric complications on clinical outcome $-\leqslant 29$ weeks.

\begin{tabular}{|c|c|c|c|c|c|}
\hline Complication & $\mathrm{N}$ & $\begin{array}{l}\text { Birth weight }(\mathrm{kg}) \\
\text { mean }( \pm \mathrm{SD})\end{array}$ & $\begin{array}{l}\text { RDS } \\
(\%)\end{array}$ & $\begin{array}{l}\text { Death } \\
(\%)\end{array}$ & $\begin{array}{l}\mathrm{PG} \\
(\%)\end{array}$ \\
\hline Blood pressure/proteinuria & 7 & $0.97(0.24)$ & 100 & 42 & 14 \\
\hline Vag. bleeding & 14 & $1.27(0.33)$ & 79 & 29 & 29 \\
\hline Premature labor & 37 & $1.14(0.32)$ & 76 & 43 & 24 \\
\hline Rupture of membranes $\geqslant 12$ hours & 13 & $1.16(0.4)$ & 69 & 54 & 77 \\
\hline Abnormal CTG & 6 & $0.97(0.28)$ & 83 & 33 & 17 \\
\hline Small for gest. age $<5^{\circ}$ & 6 & $0.71(0.21)$ & 50 & 66 & 0 \\
\hline
\end{tabular}

$\mathrm{PG}=$ Phosphatidylglycerol in all the tables

Table III. Effect of obstetric complications on clinical outcome $-30-33$ weeks.

\begin{tabular}{llllll}
\hline Complication & $\mathrm{N}$ & $\begin{array}{l}\text { Birth weight (kg) } \\
\text { mean }( \pm \text { SD) }\end{array}$ & $\begin{array}{l}\text { RDS } \\
(\%)\end{array}$ & $\begin{array}{l}\text { Death } \\
(\%)\end{array}$ & $\begin{array}{l}\text { PG } \\
(\%)\end{array}$ \\
\hline Blood pressure/proteinuria & 14 & $1.31(0.51)$ & 50 & 14 & 57 \\
Vag. bleeding & 18 & $1.85(0.3)$ & 33 & 0 & 67 \\
Premature labor & 49 & $1.76(0.34)$ & 35 & 4 & 65 \\
Rupture of membranes $\geqslant 12$ hours & 13 & $1.75(0.33)$ & 30 & 0 & 85 \\
Abnormal CTG & 9 & $1.29(0.56)$ & 44 & 0 & 33 \\
Small for gest. age $<5^{\circ}$ & 10 & $1.02(0.17)$ & 50 & 0 & 60 \\
\hline
\end{tabular}


Tables V, VI and VII relate the complications to arterial cord blood biochemistry. Again there were no obviously significant changes but it was apparent that very large standard deviations existed, particularly for the catecholamines. Under these circumstances the numbers involved are probably too small to enable firm conclusions to be drawn.

Table IV. Effect of obstetric complications on clinical outcome $-34,35$ weeks.

\begin{tabular}{lrllrl}
\hline Complication & $\mathrm{N}$ & $\begin{array}{l}\text { Birth weight }(\mathrm{kg}) \\
\text { mean }( \pm \mathrm{SD})\end{array}$ & $\begin{array}{l}\text { RDS } \\
(\%)\end{array}$ & $\begin{array}{l}\text { Death } \\
(\%)\end{array}$ & $\begin{array}{l}\text { PG } \\
(\%)\end{array}$ \\
\hline Blood pressure/proteinuria & 13 & $1.99(0.48)$ & 15 & 0 & 92 \\
Vag. bleeding & 6 & $2.53(0.38)$ & 33 & 17 & 50 \\
Premature labor & 45 & $2.23(0.34)$ & 16 & 2 & 80 \\
Rupture of membranes $\geqslant 12$ hours & 13 & $2.27(0.4)$ & 0 & 0 & 77 \\
Abnormal CTG & 7 & $1.8(0.46)$ & 14 & 28 & 57 \\
Small for gest. age $<5^{\circ}$ & 16 & $1.67(0.34)$ & 13 & 13 & 81 \\
\hline
\end{tabular}

Table V. Effect of obstetric complications on cord artery biochemistry $-\leqslant 29$ weeks; mean ( \pm SD).

\begin{tabular}{lllll}
\hline Complication & $\begin{array}{l}\text { Cortisol } \\
(\mathrm{mmol} / \mathrm{l})\end{array}$ & $\begin{array}{l}\text { Noradr. } \\
(\mathrm{nmol} / \mathrm{l})\end{array}$ & $\begin{array}{l}\text { ADR } \\
(\mathrm{nmol} / \mathrm{l})\end{array}$ & $\mathrm{pH}$ \\
\hline Blood pressure/proteinuria & $215(68)$ & $5.3(7.9)$ & $0.7(0.5)$ & $7.2(0.09)$ \\
Vag. bleeding & $170(98.9)$ & $10.1(15.4)$ & $1.4(1.6)$ & $7.18(0.18)$ \\
Premature labor & $208(134)$ & $15.1(15.5)$ & $1.6(1.6)$ & $7.22(0.12)$ \\
Rupture of membranes $\geqslant 12$ hours & $280(178)$ & $16.5(10.6)$ & $2.1(2.5)$ & $7.26(0.05)$ \\
Abnormal CTG & $253(75)$ & $7.6(6.1)$ & $1.7(1.4)$ & $7.03(0.14)$ \\
Small for gest. age $<5^{\circ}$ & $167(179)$ & $4.0(3.1)$ & $0.75(0.44)$ & $7.27(0.07)$ \\
\hline
\end{tabular}

Table VI. Effect of obstretric complications on cord artery biochemistry - 30-33 weeks; mean ( \pm SD).

\begin{tabular}{lllll}
\hline Complication & $\begin{array}{l}\text { Cortisol } \\
(\mathrm{mmol} / \mathrm{l})\end{array}$ & $\begin{array}{l}\text { Noradr. } \\
(\mathrm{nmol} / \mathrm{l})\end{array}$ & $\begin{array}{l}\text { ADR } \\
(\mathrm{nmol} / \mathrm{l})\end{array}$ & $\mathrm{pH}$ \\
\hline Blood pressure/preoteinuria & $223(92)$ & $13.6(16.0)$ & $3.1(2.9)$ & $7.21(0.1)$ \\
Vag. bleeding & $195(125)$ & $7.1(6.4)$ & $1.4(1.3)$ & $7.28(0.06)$ \\
Premature labor & $217(136)$ & $21.9(52)$ & $1.9(1.9)$ & $7.29(0.7)$ \\
Rupture of membranes $\geqslant 12$ hours & $302(85)$ & $16.1(17.7)$ & $2.6(3.2)$ & $7.28(0.1)$ \\
Abnormal CTG & $281(93)$ & $19.7(29.5)$ & $2.5(2.1)$ & $7.14(0.14)$ \\
Small for gest. age $<5^{\circ}$ & & $39(69)$ & $1.9(1.4)$ & $7.2(0.06)$ \\
\hline
\end{tabular}

Table VII. Effect of obstetric complications on arterial cord blood biochemistry - 34, 35 weeks; mean ( \pm SD).

\begin{tabular}{lllll}
\hline Complication & $\begin{array}{l}\text { Cortisol } \\
(\mathrm{mmol} / \mathrm{l})\end{array}$ & $\begin{array}{l}\text { Noradr. } \\
(\mathrm{nmol} / \mathrm{l})\end{array}$ & $\begin{array}{l}\text { ADR } \\
(\mathrm{nmol} / \mathrm{l})\end{array}$ & pH \\
\hline Blood pressure/proteinuria & $226(63)$ & $16.3(20.7)$ & $4.5(8.6)$ & $7.28(0.05)$ \\
Vag. bleeding & $310(140)$ & $5.9(2.9)$ & $1.7(0.9)$ & $7.27(0.15)$ \\
Premature labor & $407(171)$ & $17.0(19.4)$ & $3.4(5.1)$ & $7.25(0.09)$ \\
Rupture of membranes $\geqslant 12$ hours & $464(146)$ & $16.5(16.3)$ & $2.4(2.2)$ & $7.26(0.05)$ \\
Abnormal CTG & $424(173)$ & $15.1(10.4)$ & $2.4(2.2)$ & $7.15(0.14)$ \\
Small for gest. age $<5^{\circ}$ & $338(149)$ & $9.8(9.8)$ & $1.7(1.7)$ & $7.21(0.11)$ \\
\hline
\end{tabular}




\section{Discussion}

The data presented here do not support the view that certain obstetric complications accelerate fetal lung maturity. There was a slight increase in the numbers of cases with PROM who showed PG in their fluids but this did not reach statistical significance. In addition there did not seem to be a relationship between the so-called stress factors and pregnancy complication.

The best predictor of outcome was the gestational age at which the baby was born. However, some very immature babies with PG in their amniotic fluid still developed severe respiratory disease underlining the difficulties of diagnosis at this gestational age when ventilatory support is commonly required. Nevertheless, those babies with PG tended to have a more benign course, with fewer complications, and this has been confirmed in a separate study [4].

Whether obstetric complications influence neonatal outcome remains debateable. Probably gestational age at the time of delivery and the baby's condition at birth are as important.

\begin{abstract}
Obstetric complications have been associated with accelerated fetal lung maturity. In a prospective study involving 189 babies born before 36 weeks no specific obstetric complication was observed to have a significant influ-
\end{abstract}

ence on neonatal respiratory function. In addition fetal stress factors did not seem to contribute to eventual outcome.

Keywords: Neonatal respiratory distress syndrome, pregnancy complications, surfactant.

\section{Zusammenfassung}

\section{Schwangerschaftsbedingte Faktoren und Erkrankungen mit Wirkung auf den Surfactant}

In der vorliegenden Studie wurde der Einfluß verschiedener geburtshilflicher Komplikationen auf die Surfactantproduktion und die Inzidenz eines RDS beim Neugeborenen untersucht. Darüber hinaus wurden die Auswirkungen dieser Komplikationen auf die Kortisol- und Katecholaminausschüttung als „Streßprodukte“ beobachtet.

Wir untersuchten 189 Kinder mit weniger als 36 Schwangerschaftswochen. Gemessen wurde die Surfactantkonzentration im Fruchtwasser und im Sekret aus dem Pharynx; in Nabelarterie und -vene wurden die Kortisolund Katecholaminkonzentration sowie die Blutgase bestimmt. Bei den Komplikationen wurden berücksichtigt: Hypertonus/Proteinurie, vaginale Blutung, Frühgeburt, vorzeitiger Blasensprung, pathologisches CTG und intrauterine Mangelentwicklung.
Es gab keinen statistisch signifikanten Zusammenhang zwischen diesen Komplikationen und einer akzelerierten Surfactantproduktion bzw. einer geringeren Inzidenz eines RDS. Neugeborene, bei denen im Fruchtwasser bzw. im Pharynxsekret Phosphatidylglycerol nachgewiesen werden konnte, hatten eine günstigere neonatale Prognose.

Wir folgern daraus, daß Schwangerschaftskomplikationen per se das neonatale Outcome anscheinend nicht wesentlich beeinflussen. Wichtig ist das Schwangerschaftsalter zum Zeitpunkt der Geburt. Der Spiegel der im Nabelschnurblut gemessenen „Streßhormone“ wies keine Beziehung zu den Schwangerschaftskomplikationen oder zur neonatalen Lungenfunktion auf. Die Studie konnte nicht klären, durch welche Mechanismen die menschliche fetale Lunge funktionell reift.

Schlüsselwörter: Neonatales RDS, Schwangerschaftskomplikationen, Surfactant.

\begin{abstract}
Résumé
Facteurs liés à la grossesse et maladies influencant le surfactant

Cette étude a été menée pour déterminer l'effet de certaines complications obstétricales sur la production de surfactant et l'incidence du syndrome de détresse respiratoire néonatale (SDR). En outre, on a observé l'effet de ces diverses complications sur les produits de la "souffrance» fœtale: cortisol et catécholamines.
\end{abstract}

189 enfants au total ont été étudiés, tous nés avant 36 semaines. On a mesuré le surfactant dans le liquide amniotique pharyngé et on a dosé le cortisol, les catécholamines et les gaz du sang dans la veine et dans l'artère ombilicale. Les complications étudiées comprenaient: hypertension artérielle, protéinurie, métrorragies, accouchement prématuré/rupture des membranes, anomalies cardiotocographiques et hypotrophie. 
Les résultats n'ont pas permis de montrer un effet significatif sur le plan statistique de ces complications ni en terme d'accélération de l'apparition du surfactant, ni en terme de diminution du SDR. Les nouveaux-nés dont le liquide amniotique pharyngé contient du phosphatidylglycérol ont un devenir néonatal moins grave.

Les conclusions de cette étude sont que les complications de la grossesse en elles-mêmes n'apparaissent pas in- fluencer significativement le devenir néonatal. Le facteur principal est l'âge gestationnel au moment de la naissance. Les taux des produits du «stress » trouvés au sang du cordon ne sont pas corrélés aux complications de la grossesse ni aux fonctions des poumons en période néonatale. Les mécanismes par lesquels le poumon du fotus human devient fonctionellement mature n'ont pas été élucidés par cette étude.

Mots-clés: Complications de la grossesse, surfactant, syndrome de détresse respiratoire néonatale.

Acknowledgements: Part of this work was supported by the Scottish Hospitals Endowment Trust (HERT613).

\section{References}

[1] Ball SG, M Tree, JJ Morton, GC Inglis, R FrASER: Circulating dopamine, catecholamines, re$\mathrm{nin}$, angiotensin, aldosterone and vasopressin in thr conscious dog. Clin Sci 61 (1982) 417

[2] DobBIE HG: Obstetric predictors of neonatal outcome. M.D. Thesis in preparation

[3] Kankare P, M SuovaniEMi: J Chromatogr 62 (1971) 485

[4] Whittle MJ, AI MacGillivray, KP Hanretty, HG DoBBIE, CA HowIE: Phosphatidylglycerol and neonatal mortality and morbidity. J Obstet Gynaecol 7 (1986) 23
[5] Whittle MJ, AI Wilson, CR Whitfield: Amniotic fluid PG - an early indication of fetal lung maturity. Br J Obstet Gynaecol 90 (1983) 134

[6] Whittle MJ, AI Wilson, CR Whitfield, RD PATON, RW LOGAN: Amniotic fluid phospholipid profile determined by CD-TLC as index of fetal lung maturity. Br Med J 282 (1981) 428

Martin J Whittle, M. D.

Consultant Obstetrician

Department of Midwifery

The Queen Mother's Hospital

Yorkhill

Glasgow G3 8SH, Scotland 


\section{Tunnessen · Spranger Symptome in cler päclatrie \\ Eine Differentialdiagnose in Stichworten}

$17 \times 24 \mathrm{~cm}$. X, 566 Seiten. 1987. Gebunden DM 128,ISBN 3110101467

Die amerikanische Originalausgabe des anerkannten Pädiaters W. Tunnessen gehört bereits wenige Jahre nach Erscheinen zu den Standardnachschlagewerken der Kinderheilkunde.

In Ergänzung pädiatrischer Lehrbücher werden in diesem Werk die wichtigsten Einzelsymptome - gegliedert nach topographischen und systematischen Gesichtspunkten - in ihrer diagnostischen und differential-diagnostischen Wertigkeit ausführlich beschrieben.

Einer einleitenden Definition des Krankheitssymptoms und seiner Abgrenzung zum Normalbefund folgen jeweils ausführlich kommentierte Listen möglicher zugrundeliegender Krankheitsbilder. Strategien des weiteren Vorgehens zur Sicherung der Diagnose werden ausführlich dargestellt. „Symptome in der Pädiatrie“ ist die von einem der namhaftesten deutschen Pädiater durchgesehene und auf die deutsche Terminologie abgestellte Übersetzung dieses Werkes.

Es ist für Kinderärzte eine hilfreiche Sammlung von „Checklists“ zum Nachschlagen im Einzelfall, für Ärzte anderer Fachrichtungen bietet es eine schnelle und gründliche Information. Für Medizinstudenten ist es darüber hinaus auch ein sinnvolles Lehrbuch der pädiatrischen Symptomatologie.

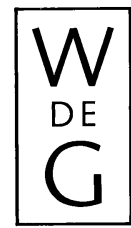

